\title{
Suppurative Salmonella thyroiditis coexistence with invasive thymoma
}

\author{
Ke-Cheng Chen, MD, ${ }^{a}$ Yih-Leong Chang, MD, ${ }^{\mathrm{b}}$ Chien-Te Pan, MD, ${ }^{a}$ and Yung-Chie Lee, MD, PhD, ${ }^{a}$ Taipei, Taiwan
}

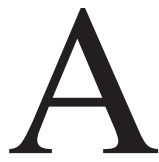

cute suppurative thyroiditis is a rare disorder. The rarity may be because the thyroid gland has a good blood supply, protective capsule, and high local iodine concentration. Acute suppurative thyroiditis caused by the Salmonella species is even rarer and is often associated with certain anatomic abnormality or immunologic defects. We report a 60-year-old woman with an underlying invasive thymoma who presented with Salmonella bacteremia and thyroiditis. The patient underwent a successful simultaneous total thymothymectomy and left subtotal thyroidectomy.

\section{Clinical Summary}

Our 60-year-old female patient had a history of hypertension, hyperlipidemia, and arrhythmia under regular medical control. One week before admission, she had fever with chills. In addition, she palpated a left neck mass with tenderness. She visited a local hospital where a computed tomographic scan showed a hypodense lesion in the left thyroid lobe (Figure 1, A) and a large mass with focal calcification in the anterior mediastinum (Figure 1, B). During the hospitalization, leukocytosis with a high level of C-reactive protein was detected. The blood culture yielded Salmonella group D1. Neither diarrhea nor abdominal pain was noted. She was then referred to our hospital for further management. On arrival, empirical antibiotics with ceftriaxone (Rocephin, Roche Laboratories, Indianapolis, Ind) for Salmonella bacteremia were given. The fever subsided, and the blood cultures and stool culture were negative. The thyroid sonogram showed bilateral multinodular goiters with a cystic change at the left lobe. After preoperative examinations were complete and the infection was under control, the patient underwent surgery for the suspicious teratoma and thyroid mass. The operation method included a median sternotomy for total excision of the mediastinal mass and collar incision for a left subtotal thyroidectomy. The operative finding showed a mediastinal mass measuring $9 \times 9 \times 5 \mathrm{~cm}$ with nodularity and a thyroid mass measuring $5 \times 3 \times 3 \mathrm{~cm}$. The pathologic examination disclosed an invasive thymoma, which was highlighted by cytokeratin stain (Figure 2, A), and thyroiditis with microabscess and adjacent neck muscle involvement (Figure 2, $B$ ). The postop-

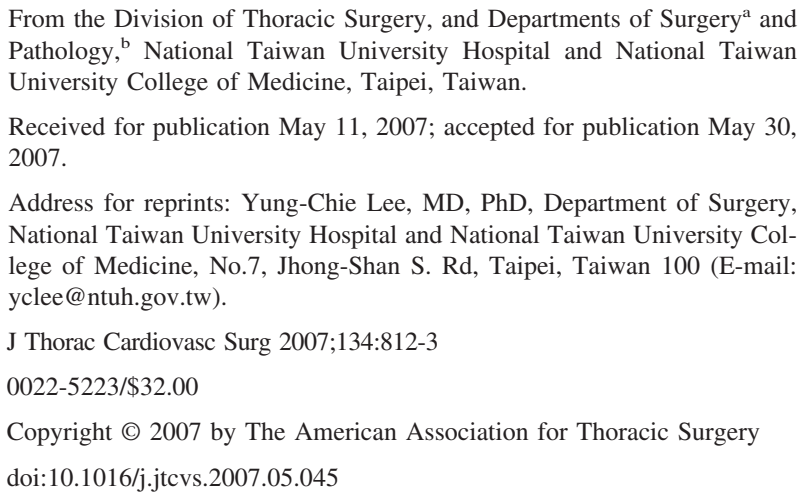

erative course was uneventful. The thyroid gland culture yielded Salmonella group D1. The patient received intravenous ceftriaxone for 2 weeks and was then administered the oral form. The patient was discharged on postoperative day 5 , and adjuvant radiotherapy for invasive thymoma was arranged in the outpatient department. Currently, she is well with no evidence of disease or infection.

\section{Discussion}

The thyroid gland is an unusual location for a suppurative process because of its anatomic position, capsule, rich system of blood and lymphatic supply, and high iodine content, which inhibit bacterial proliferation. According to the studies of Berger and colleagues, ${ }^{1}$ most causative pathogens of suppurative thyroiditis are grampositive bacteria such as streptococci and staphylococci. Acute suppurative thyroiditis is a progressive disease and is potentially fatal if it is not diagnosed rapidly and treated properly. Most of the suppurative thyroiditis contributes from the extension of adjacent structures, trauma with direct inoculation, or hematogenous
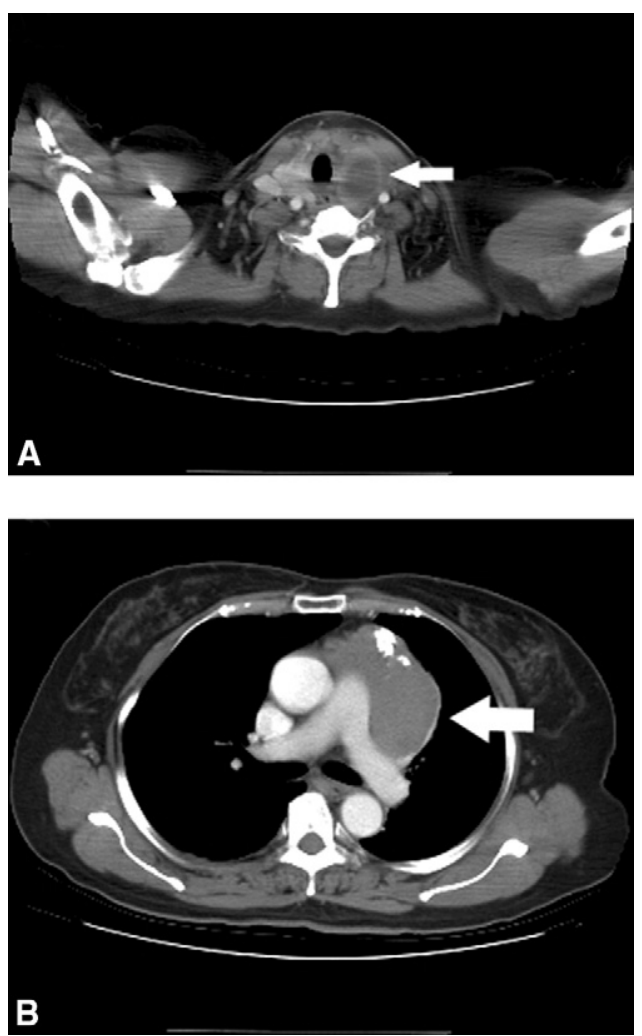

Figure 1. A, Chest computed tomographic scan of a left thyroid mass with hypodense lesion (arrow). B, The same chest computed tomography showed a large mediastinal mass with focal calcification. The tumor infiltration to adjacent structures was demonstrated (arrow). 

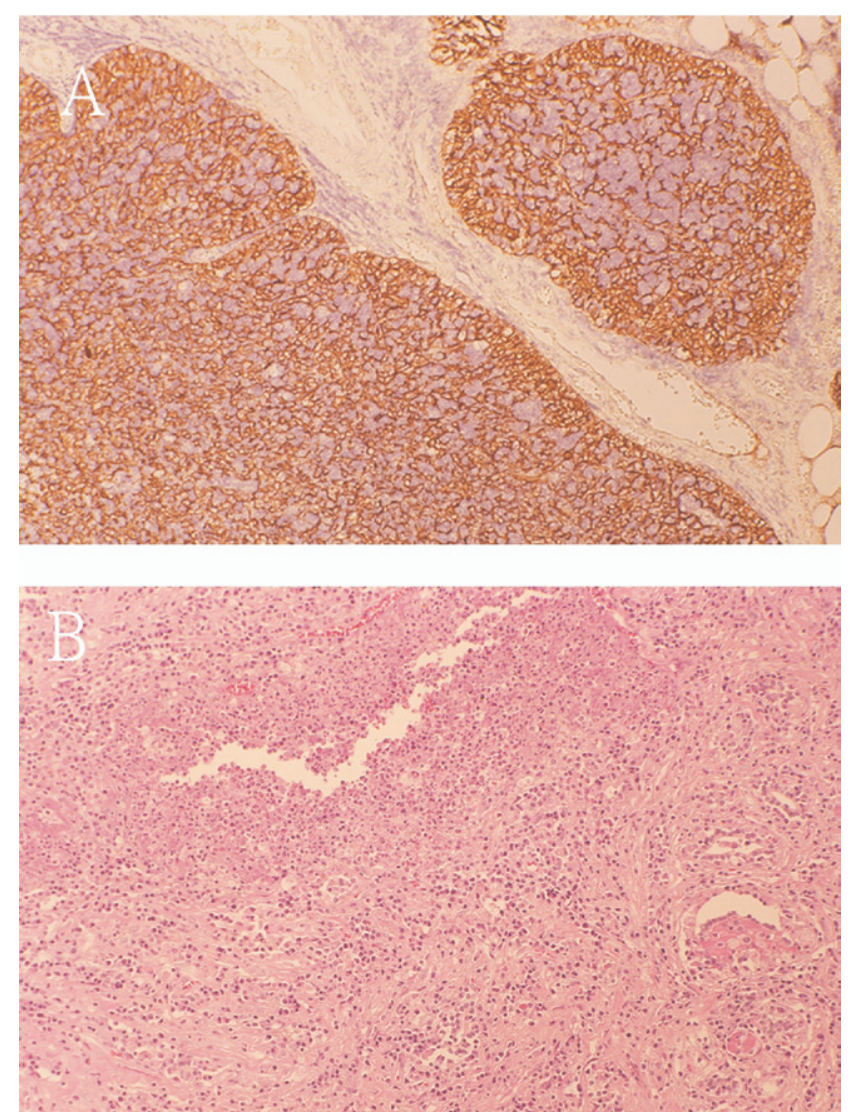

Figure 2. A, The left anterior mediastinal mass demonstrated a thymoma with extracapsular invasion highlighted by cytokeratin stain (33× magnification); $B$, The left thyroid hypodense area was replaced by acute and chronic inflammation with microabscess formation (hematoxylin-eosin, 33× magnification).

spreading from a distant focus. Thyroiditis is reported to be associated with $0.5 \%$ to $5 \%$ of patients with thymoma. ${ }^{2,3}$ Patients with suppurative Salmonella thyroiditis are often reported to have had thyroid disease, certain anatomic abnormalities, immunologic disturbances, or malignancies that can contribute to reduce resistance to infection. ${ }^{4}$ Our patient had an extensive medical history but undiagnosed multinodular goiter and invasive thymoma, which thus posed an increased risk for the development of a localized abscess during bacteremia. There was no pyriform sinus fistula noted in the computed tomography, which was considered to be the most common route of thyroidal infection. ${ }^{5}$ Despite our efforts, the source and route of primary infection remained unclear. The hematogenous route seems to be the most plausible for the patient's local abscess, although the stool culture was negative. Extraintestinal manifestation of Salmonella infection is not rare, and any organ can be involved. Unusual local symptoms of thyroid goiter, such as anterior neck pain or fever that is suspected to be a local infection in an immunocompromised host or underlying malignancy, should be promptly investigated. Timely administration of appropriate antibiotics and surgical intervention can reduce serious complications.

\section{Conclusions}

The immunocompromised status of our patient was caused by the coexistent invasive thymoma, which may have predisposed her to the Salmonella bacteremia from an unknown cause. The hematogenous spreading to the multinodular goiter then contributed to the abscess formation in the left lobe. A total thymothymectomy and left subtotal thyroidectomy were performed. The patient recovered and was discharged with no sign of infection. To our knowledge, this is the first case report of successful 1-stage surgical management of invasive thymoma and Salmonella thyroiditis.

\section{References}

1. Berger SA, Zonszein J, Villamena P, Mittman N. Infectious diseases of the thyroid gland. Rev Infect Dis. 1983;5:108-22.

2. Verley JM, Hollmann KH. Thymoma. A comparative study of clinical stages, histologic features, and survival in 200 cases. Cancer. 1985;55: 1074-86.

3. Souadjian JV, Enriquez P, Silverstein MN, Pepin JM. The spectrum of diseases associated with thymoma. Coincidence or syndrome? Arch Intern Med. 1974;134:374-9.

4. Su DH, Huang TS. Acute suppurative thyroiditis caused by Salmonella typhimurium: a case report and review of the literature. Thyroid. 2002; 12:1023-7.

5. Miyauchi A, Matsuzuka F, Kuma K, Takai S. Piriform sinus fistula: an underlying abnormality common in patients with acute suppurative thyroiditis. World J Surg. 1990;14:400-5. 